\title{
Rancidity Estimation of Perilla Seed Oil by Using Near-Infrared Spectroscopy and Multivariate Analysis Techniques
}

\author{
Suk-Ju Hong, ${ }^{1}$ Shin-Joung Rho, ${ }^{1}$ Ah-Yeong Lee, ${ }^{1}$ Heesoo Park, ${ }^{1}$ Jinshi Cui, ${ }^{1}$ Jongmin Park, ${ }^{2}$ \\ Soon-Jung Hong, ${ }^{3}$ Yong-Ro Kim, ${ }^{1}$ and Ghiseok Kim ${ }^{1,4}$ \\ ${ }^{1}$ Department of Biosystems and Biomaterials Science and Engineering, Seoul National University, 1 Gwanak-ro, Gwanak-gu, \\ Seoul 08826, Republic of Korea \\ ${ }^{2}$ Department of Bio-Industrial Machinery Engineering, Pusan National University, 1268-50 Samnangjin-ro, Cheonghak-ri, \\ Samnangjin-eup, Miryang-si 50463, Republic of Korea \\ ${ }^{3}$ Rural Human Resource Development Center, Rural Development Administration, 420 Nongsaengmyeong-ro, Wansan-gu, \\ Jeonju-si 54874, Republic of Korea \\ ${ }^{4}$ Research Institute of Agriculture and Life Sciences, Seoul National University, 1 Gwanak-ro, Gwanak-gu, Seoul 08826, \\ Republic of Korea
}

Correspondence should be addressed to Ghiseok Kim; ghiseok@snu.ac.kr

Received 12 April 2017; Revised 10 July 2017; Accepted 19 July 2017; Published 17 September 2017

Academic Editor: Jose S. Camara

Copyright (C) 2017 Suk-Ju Hong et al. This is an open access article distributed under the Creative Commons Attribution License, which permits unrestricted use, distribution, and reproduction in any medium, provided the original work is properly cited.

\begin{abstract}
Near-infrared spectroscopy and multivariate analysis techniques were employed to nondestructively evaluate the rancidity of perilla seed oil by developing prediction models for the acid and peroxide values. The acid, peroxide value, and transmittance spectra of perilla seed oil stored in two different environments for 96 and $144 \mathrm{~h}$ were obtained and used to develop prediction models for different storage conditions and time periods. Preprocessing methods were applied to the transmittance spectra of perilla seed oil, and multivariate analysis techniques, such as principal component regression (PCR), partial least squares regression (PLSR), and artificial neural network (ANN) modeling, were employed to develop the models. Titration analysis shows that the free fatty acids in an oil oxidation process were more affected by relative humidity than temperature, whereas peroxides in an oil oxidation process were more significantly affected by temperature than relative humidity for the two different environments in this study. Also, the prediction results of ANN models for both acid and peroxide values were the highest among the developed models. These results suggest that the proposed near-infrared spectroscopy technique with multivariate analysis can be used for the nondestructive evaluation of the rancidity of perilla seed oil, especially the acid and peroxide values.
\end{abstract}

\section{Introduction}

Recently, westernization and gentrification of food has increased the variety of processed food, and the expanding consumption pattern aimed at pursuing wellbeing and health is increasing the interest and demand in healthy highfunctioning vegetable oils. Also, with the advent of problems, such as obesity and adult diseases, caused by trans fats and cholesterol from vegetable oils like soybean oil and corn oil, which were previously extensively used, the demand for high-quality vegetable oils, such as olive oil and grape seed oil, is increasing; recently, the interest in premium vegetable oils, for example, canola oil, green tea oil, and brown rice oil, has rapidly increased. Oils are known to be not only a high-energy source, as one of the three major nutrient groups of carbohydrates, proteins, and oils, but also an important and useful component of the human body; it is present in cell membranes as a fat-soluble carrier and also protects hypodermic tissues and organs [1]. In particular, perilla seed oil, a traditional Korean vegetable oil, contains $60 \%$ linolenic acid, one of the essential fatty acids, which is known to be useful in brain and nervous tissue development and in adult disease prevention. Generally, perilla (Perilla frutescens Britton var. japonica Hara) is an annual plant belonging to the family Labiatae. Its native habitat is Southeast Asia and it is commonly found in Korea, China, India, and Japan. 
Records indicate that it was raised approximately 1300 years ago in Korea along with sesame, and it grows easily in every area of a mountain or roadside swamp to be raised on a national scale [2-4].

Note that the smell, fragrance, and taste of vegetable oils used in most homes, restaurants, and the food processing industry tends to change because of the various chemical and microbial factors during storage and processing. This decreases its nutritive value, which in turn deteriorates quality as well as that of the processed foods, and sometimes, toxic agents that are harmful to humans are also generated. This oil deterioration is called rancidity $[5,6]$. Oil deterioration is known to occur by various causes, including autooxidation if the oil component absorbs oxygen. Autooxidation of vegetable oil increases its weight and causes the formation of aldehydes and ketones. Vegetable oil oxidation caused by heating at high temperatures increases the content of free fatty acids, lowers the smoke point, and leads to bubbling; acrolein, which is harmful to humans, may also form. In particular, vegetable oils containing a large amount of unsaturated fatty acids and, if heated for a long time and repetitively, may generate the toxic chemical compound 4hydroxy-trans-2-nonenal, which can cause cardiovascular disorders [7-10].

As an existing method to measure oil rancidity, physicochemical titration is widely used to measure the acid value, peroxide value, and so forth. However, physicochemical titration analysis may generate errors resulting from the experimenter's skills and is expensive and time-consuming and hence is not suitable for repetitive experiments. Therefore, the development of a technique to nondestructively analyze vegetable oil rancidity in real time is attracting much interest. Recently, a study has been conducted into spectroscopy to nondestructively analyze and predict variation in the components and quality of agricultural products or food. Near-infrared (NIR) spectroscopy can obtain a signal of relatively high energy, relative to far-infrared radiation and microwaves, and can detect a particular spectrum of an intrinsic component of a test object during wavelength bandwidth measurement. Its relatively simple device composition of sensor and light source makes it easy to implement; therefore, NIR spectroscopy is being widely used in researches into the nondestructive quality analysis of agricultural products and food $[11,12]$.

Generally, near-infrared rays have a high energy level relative to mid- and far-infrared rays, and hence, they have low optical absorbance on test samples and excellent penetrability; this means that the technique is little influenced by test sample thickness and there is no need for the preprocessing of the test sample. Also, the components of near-infrared ray light splitters, such as fiber-optic cables, monochromators, and detectors, are reported to be easy to set up and operate relative to mid-infrared ray light splitters and have excellent measurement repeatability. In particular, fiberoptic communications have been developed based on nearinfrared rays; consequently, their use makes it possible to measure and analyze spectra remotely in real time and allows the development of telemetering device. Therefore, nearinfrared rays can be used for the composition of real-time analysis devices for material process control and quality analysis. In spite of the many merits of NIR spectroscopy, the measurement environment and instruments may cause noise in the NIR spectra, which may lead to the degradation of spectrum analysis. Therefore, it is very important to perform appropriate preprocessing methods to remove these noise components and to develop a suitable optimal model to analyze and predict sample components by using spectra data. Until now, NIR spectroscopy research on agricultural products has been applied to the internal quality analysis of fruits and vegetables by using multivariate analysis methods, such as PLSR (partial least square regression), PCR (principal component regression), and MLR (multiple linear regression) [13-18]. In addition, during the 2000s, artificial neural network (ANN) techniques have been steadily applied to the internal quality analysis of food and agricultural products with visible/NIR spectrum analysis, and various kinds of research have been conducted on production date range distinction of milk products and component analysis and quality prediction for fruit juice, wine, and so forth [19-22].

This study employed NIR spectroscopy and multivariate analysis techniques to nondestructively discern the rancidity of perilla seed oil, in conjunction with the storage conditions, and to evaluate the effectiveness of the analysis methods. For this purpose, perilla seed oil was stored in specific environments for a certain period of time, and the acid value, peroxide value, and transmittance spectra of the same perilla seed oil were measured by physicochemical titration analysis and NIR spectroscopy. We used PCR, PLSR, and ANN analysis methods to quantitatively predict the rancidity extent of perilla seed oil from the collected data, and then we evaluated the performance of each model in conformity with the applied preprocessing methods.

\section{Material and Methods}

2.1. Sample Materials. Perilla (Perilla frutescens Britton var. japonica Hara) seeds harvested in October 2016 were acquired twice from a local producer (Miryang, South Korea) with a one-week interval to prepare two a little different sample groups, and they were cleaned with water and air-dried to remove moisture. After drying, the perilla seeds were roasted for $60 \mathrm{~min}$ at $200^{\circ} \mathrm{C}$, and the heated perilla seeds were squeezed for $15 \mathrm{~min}$ with a pressure of $600 \mathrm{kgf} / \mathrm{cm}^{2}$ by using an oil press to extract the perilla seed oil. The extracted perilla seed oil was kept in an air-tight opaque container under refrigeration at $5^{\circ} \mathrm{C}$ before the experiments. For our test, perilla seed oil samples were separated into two groups and stored in two different environments to artificially accelerate rancidity. The first group was stored at a controlled temperature of $60^{\circ} \mathrm{C}$ with a relative humidity of $40 \%$ for $4 \mathrm{~d}(96 \mathrm{~h})$, and the second sample group was stored for $6 \mathrm{~d}(144 \mathrm{~h})$ at a higher temperature of $80^{\circ} \mathrm{C}$ with a lower relative humidity of $10 \%$.

2.2. Determination of Acid Value. The acid value (AV) of the samples was determined by the method of the American Oil Chemists' Society (1977) with some modifications [23]. One gram of each sample was dissolved in $20 \mathrm{~mL}$ of ether : ethanol 
$(2: 1, v / v)$ solution, and then, $1 \%(v / v)$ phenolphthalein solution was added as an indicator. The mixture was agitated and titrated with $0.1 \mathrm{~N} \mathrm{KOH}$ solution until the appearance of a pink color. Titration analysis was also performed for a blank sample under the same conditions. The AV was expressed as milligrams of $\mathrm{KOH}$ per gram of oil $(\mathrm{mg} / \mathrm{g})$ and determined according to the following equation.

$$
\mathrm{AV}=\frac{5.611 \times A \times F}{W},
$$

where AV is the acid value, $A$ denotes the volume of $\mathrm{KOH}$ $(\mathrm{mL}), 5.611$ is the constant value equivalence of mass of $0.1 \mathrm{~N} \mathrm{KOH}, W$ is the weight of sample, and $F$ is the normality of the standard $\mathrm{KOH}$ solution.

2.3. Determination of Peroxide Value. The peroxide value $(\mathrm{PV})$ is a measure of the peroxides contained in the oil during storage. The PV of the oil was measured by iodine released from potassium iodide (KI) according to the method of the Association of Analytical Communities (1995) with slight modifications [24]. One gram of each sample was mixed with $25 \mathrm{~mL}$ of chloroform : acetic acid $(2: 3, v / v)$ solution and $1 \mathrm{~mL}$ of saturated KI solution with stirring and kept in a dark place for $5 \mathrm{~min}$. Subsequently, the solution was mixed with $30 \mathrm{~mL}$ of distilled water and $1 \%(w / v)$ starch solution as an indicator. The mixture was titrated with $0.01 \mathrm{~N} \mathrm{Na}_{2} \mathrm{~S}_{2} \mathrm{O}_{3}$ until the color of the reactant completely disappeared. The same preparation without an oil sample served as a blank experiment. The PV was represented as milliequivalents of oxygen per kilogram of oil $(\mathrm{meq} / \mathrm{kg})$ and was calculated by the following equation.

$$
\mathrm{PV}=\frac{(A-B) \times F \times 100}{W},
$$

where $\mathrm{PV}$ is the peroxide value, $A$ and $B$ denote the volume of $\mathrm{Na}_{2} \mathrm{~S}_{2} \mathrm{O}_{3}(\mathrm{~mL})$ in the sample and blank, respectively, $W$ is the weight of sample, and $F$ is the normality of the standard $\mathrm{Na}_{2} \mathrm{~S}_{2} \mathrm{O}_{3}$ solution.

2.4. Measurement of NIR Transmittance Spectra. As illustrated in Figure 1, the transmittance spectra of perilla seed oil samples were measured by using a NIR spectrometer (CDI-NIR128, Control Development Inc., USA), with a wavelength range from 900 to $1700 \mathrm{~nm}$, and a tungstenhalogen lamp (LS-1, Ocean optics, USA) was used as the light source.

Perilla seed oil samples were separated into two groups and stored in two different environments. The first sample group was stored at a controlled temperature of $60^{\circ} \mathrm{C}$ with a relative humidity of $40 \%$ for $4 \mathrm{~d}(96 \mathrm{~h})$ to accelerate rancidity. Samples were taken at time zero, and then $10 \mathrm{~g}$ of 12 oil samples were collected every $24 \mathrm{~h}$ in order to obtain transmittance spectra after different periods. Therefore, we acquired 60 spectra for the first perilla seed oil sample group in $4 \mathrm{~d}$ (96h). The environmental conditions for the second sample group were similar but employed higher temperature and lower relative humidity, that is, the temperature and relative humidity of the storage chamber were controlled as $80^{\circ} \mathrm{C}$, $10 \%$ for $6 \mathrm{~d}(144 \mathrm{~h})$, respectively. Samples were again taken

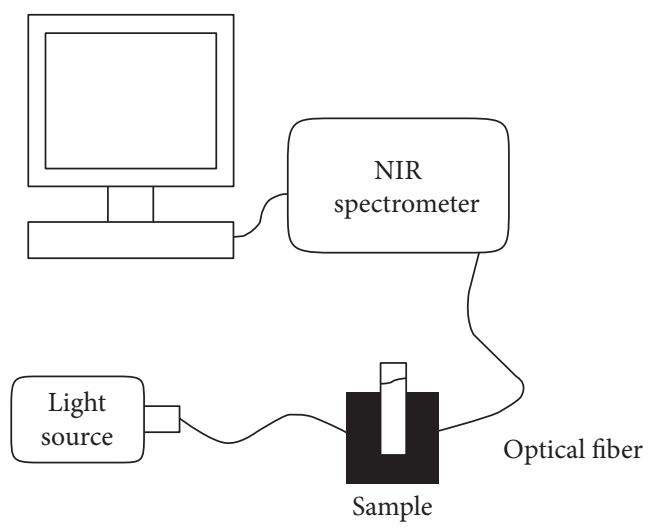

FIgURE 1: Schematic representation of the NIR spectra measuring instrument.

at time zero, and then in this case, $10 \mathrm{~g}$ of nine perilla seed oil samples were collected every $24 \mathrm{~h}$ to obtain transmittance spectra after different periods. Therefore, we acquired 63 spectra for the second perilla seed oil sample group in $6 \mathrm{~d}$ $(144 \mathrm{~h})$. At the end, we had prepared 123 spectra for the two different groups of perilla seed oil samples. All spectra were prepared with ten replicates at each measuring point with $50 \mathrm{~ms}$ of measuring exposure time.

The transmittance of measured spectra was calibrated by using both white-referenced and dark-referenced spectra as shown in (3). The white-referenced spectrum was measured from the transmittance through the blank cuvette used in the test, and the dark-referenced spectrum measured with the fiber optic cable was completely blocked.

$$
\text { Transmittance }(\%)=\frac{S_{i}-D}{R-D} \times 100,
$$

where $S_{i}$ is the raw transmittance of the samples and $D$ and $R$ represent the raw transmittance of the dark and white referenced spectra, respectively.

2.5. Model Development by Using Multivariate Analysis. Light scattering, optical path changes, and noise can be induced in the obtained transmittance spectra from the measuring environment and instruments; therefore, preprocessing methods should be applied to the measured spectra in order to remove noise components and correct the spectra. In this study, we employed scatter correction techniques, such as MSC (multiplicative scatter correction) and SNV (standard normal variate), and normalization techniques, including maximum normalization, mean normalization, and range normalization, as preprocessing methods.

We used the PCR and PLSR methods that are defined as (4) to predict the acid value and peroxide value, which can be used to estimate the extent of oil rancidity, according to storage conditions and periods. The performances of the developed models are compared and analyzed according to the applied preprocessing methods. Among the obtained 123 spectra, calibration and validation modeling operations used 82 and 41 spectra, respectively. Algorithms for preprocessing and regression modeling were developed by 


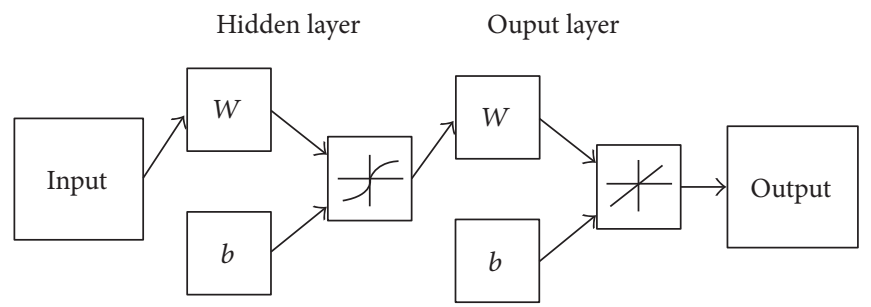

FIGURE 2: Schematic structure of the ANN model.

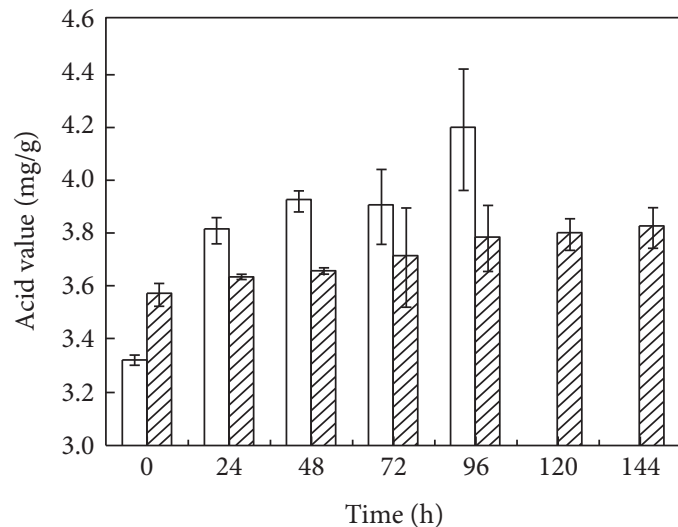

$\square$ Storage temp. $60^{\circ} \mathrm{C}$ with $40 \% \mathrm{RH}$

ZIA Storage temp. $80^{\circ} \mathrm{C}$ with $10 \% \mathrm{RH}$

(a)

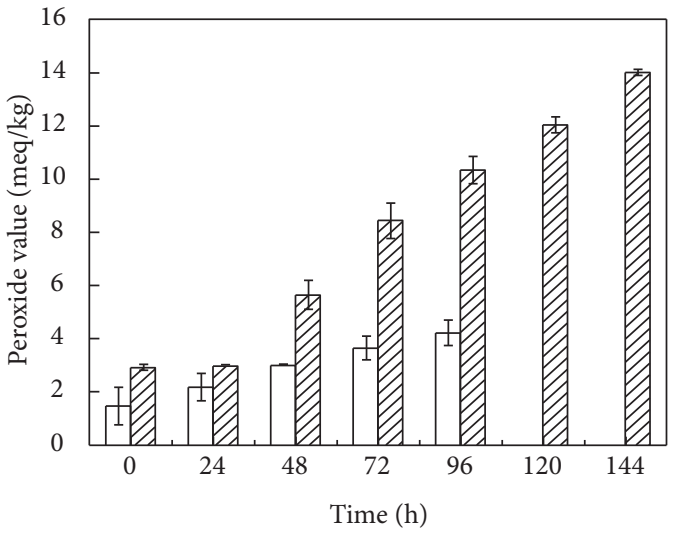

Storage temp. $60^{\circ} \mathrm{C}$ with $40 \% \mathrm{RH}$

$\mathrm{ZZ}$ Storage temp. $80^{\circ} \mathrm{C}$ with $10 \% \mathrm{RH}$

(b)

Figure 3: Acid values and peroxide values of perilla seed oil for various storage temperatures, relative humidity, and time periods. (a) Acid value. (b) Peroxide value.

TABLE 1: Statistics of acid values and peroxide values of perilla seed oil according to storage time.

\begin{tabular}{lcccccccc}
\hline \multirow{2}{*}{ Measurements } & \multirow{2}{*}{ Storage conditions } & 0 & 24 & 48 & Time (h) & 72 & 96 & 120 \\
\hline \multirow{2}{*}{ Acid value $(\mathrm{mg} / \mathrm{g})$} & $60^{\circ} \mathrm{C}, 40 \% \mathrm{RH}$ & $3.32 \pm 0.02$ & $3.81 \pm 0.05$ & $3.92 \pm 0.04$ & $3.9 \pm 0.14$ & $4.19 \pm 0.23$ & - & - \\
& $80^{\circ} \mathrm{C}, 10 \% \mathrm{RH}$ & $3.57 \pm 0.04$ & $3.63 \pm 0.01$ & $3.65 \pm 0.01$ & $3.71 \pm 0.19$ & $3.78 \pm 0.13$ & $3.80 \pm 0.06$ & $3.82 \pm 0.08$ \\
Peroxide value $(\mathrm{meq} / \mathrm{kg})$ & $60^{\circ} \mathrm{C}, 40 \% \mathrm{RH}$ & $1.48 \pm 0.68$ & $2.18 \pm 0.50$ & $2.99 \pm 0.30$ & $3.65 \pm 0.45$ & $4.22 \pm 0.45$ & - & - \\
& $80^{\circ} \mathrm{C}, 10 \% \mathrm{RH}$ & $2.91 \pm 0.10$ & $2.98 \pm 0.38$ & $5.64 \pm 0.54$ & $8.45 \pm 0.66$ & $10.33 \pm 0.50$ & $12.03 \pm 0.29$ & $14.00 \pm 0.11$ \\
\hline
\end{tabular}

using commercial software (Matlab ver. R2016, MathWorks, Natick, MA, USA).

$$
\begin{aligned}
& X=T P^{T}+E, \\
& Y=T Q^{T}+F,
\end{aligned}
$$

where $X$ is the $n$ by $m$ matrix of predictors, $Y$ is the $n$ by $p$ matrix of responses, $T$ is the $n$ by 1 matrix of the score matrix, $E$ and $F$ are error terms, and $P$ and $Q$ are the $m$ by 1 and $p$ by 1 loading matrices, respectively.

In the development process of both PCR and PLSR models, the selection of the number of PCs (principal components) should be significantly considered. If the number of PCs is too small, the regression model becomes inaccurate because the complete measurement information cannot be sufficiently reflected in the developing model. By contrast, overfitting of the regression model, which may reduce its performance, can be induced if a large number of PCs are selected. The performance of the PCR and PLSR models that were developed to estimate the extent of rancidity was assessed with the RMSE (root-mean-square error), as defined in (5). The regression model with a small RMSE and large coefficient of determination was selected as the appropriate model.

$$
\mathrm{RMSE}=\sqrt{\frac{\sum_{i=1}^{m}\left(y_{\mathrm{obs}}-y_{\mathrm{pre}}\right)^{2}}{m}},
$$

where RMSE represents the sample standard deviation of the differences between predicted values and observed values. 


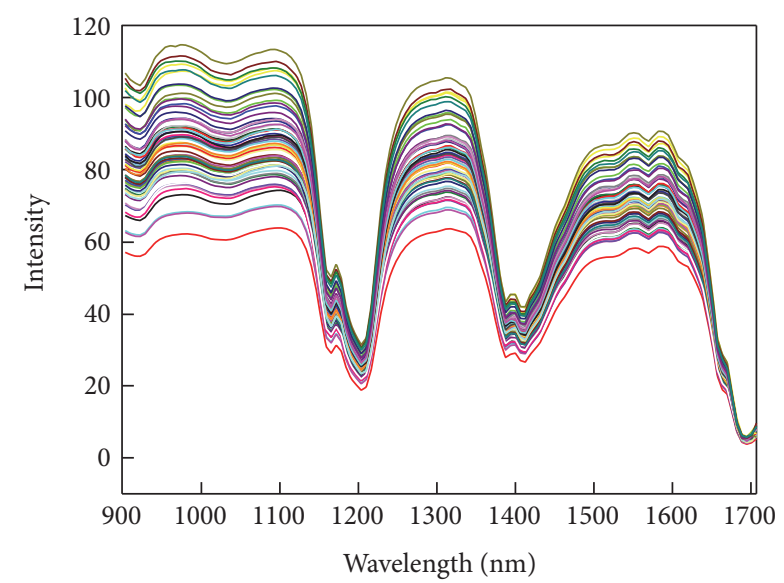

FIGURE 4: Transmittance spectra of perilla seed oil samples according to storage time.

$y_{\mathrm{obs}}$ and $y_{\text {pre }}$ are the observed and predicted values, respectively. $m$ is the number of data.

We also developed ANN-based regression models that can predict the acid and peroxide values by using NIR transmittance spectra obtained from perilla seed oil samples. As shown in Figure 2, the models fundamentally consisted of three parts, namely, input, hidden, and output layers, and both supervised-learning method and LevenbergMarquardt back-propagation algorithms were used in this study. The operating system, CPU, and RAM capacity that were used for the development of the ANN-based regression models were OS X El Capitan, $2.6 \mathrm{GHz}$ Intel Core $\mathrm{i} 5$, and $8 \mathrm{~GB}$, respectively. The algorithms needed for the ANN process were created by using commercial software (Matlab ver. R2016, MathWorks, Natick, MA, USA). For the development of the ANN-based models, the NIR transmittance spectra of perilla seed oil samples were set to input (dependent variable) and both acid and peroxide values were set to output (independent variable). We designed four kinds of hidden layers by selecting different node numbers as 50, 70, 90, and 110. In addition, a sigmoid function was used as the activation function, and the iteration was limited to a maximum of 1000 to prevent overflow during calculations. Among 123 spectra $(100 \%)$, the ANN operations of training, validation, and testing used $73(60 \%), 25(20 \%)$, and 25 spectra $(20 \%)$, respectively.

\section{Results and Discussion}

3.1. Titration Analysis of Perilla Seed Oil. Oil oxidation, the main cause of rancidity, is the process by which the double bonds of unsaturated fatty acids in the oil combine with oxygen in the air to form oxidation products, with accompanying off-flavors and smells. The AVs and PVs measured in this study are widely used as key indicators of the extent to which rancidity reactions have occurred during the initial and intermediate stages of oil oxidation. They could be used as an indication of the quality and stability of oils. Generally, the more the oil rancidity has progressed, the more these
TABLE 2: Locations of function groups and corresponding vibration modes in the near-infrared region.

\begin{tabular}{lcc}
\hline Wavelength $(\mathrm{nm})$ & \multicolumn{1}{c}{ Vibration mode } & Structure \\
\hline 900 & C-H str. third overtone & $\mathrm{CH}_{3}$ \\
1195 & C-H str. second overtone & $\mathrm{CH}_{3}$ \\
1215 & C-H str. second overtone & $\mathrm{CH}_{3}$ \\
1395 & 2C-H str. +2C-H def. & $\mathrm{CH}_{3}$ \\
1410 & O-H str. first overtone & $\mathrm{ROH}$ \\
\hline
\end{tabular}

values have increased $[25,26]$. The extent of rancidity of the perilla seed oil according to the storage environment and period was measured as the AV and PV, and the results are summarized in Figure 3 and Table 1. The oils were stored at two different conditions of temperature and relative humidity (condition 1: $60^{\circ} \mathrm{C}$ with $40 \% \mathrm{RH}$ and condition 2 : $80^{\circ} \mathrm{C}$ with $\left.10 \% \mathrm{RH}\right)$. There were significant rises in the $\mathrm{AVs}$ and $\mathrm{PVs}$ under all conditions during the storage period. The AVs of oil samples stored for $4 \mathrm{~d}(96 \mathrm{~h})$ under conditions 1 and 2 were $4.19 \pm 0.23 \mathrm{mg} / \mathrm{g}$ and $3.78 \pm 0.13 \mathrm{mg} / \mathrm{g}$, respectively, and the PVs were $4.22 \pm 0.45 \mathrm{meq} / \mathrm{kg}$ and $10.33 \pm 0.5 \mathrm{meq} / \mathrm{kg}$, respectively.

From the incremental results of AVs and PVs that were obtained during the initial and intermediate processes of oil oxidation under the two storage conditions, it can be observed that the AV of the oil sample stored under condition $1\left(60^{\circ} \mathrm{C}, 40 \% \mathrm{RH}\right)$ was higher than that of the oil sample stored under condition $2\left(80^{\circ} \mathrm{C}, 10 \% \mathrm{RH}\right)$. That is, the AV of the oil sample stored at the lower relative humidity of $10 \%$ was generally stabilized during the six days at under $3.82 \mathrm{mg} / \mathrm{g}$, whereas the observed AV at the higher relative humidity of $40 \%$ increased continuously during the four days until it reached $4.19 \mathrm{mg} / \mathrm{g}$. In contrast to the $\mathrm{AV}$, the $\mathrm{PV}$ of the oil sample stored under condition $2\left(80^{\circ} \mathrm{C}, 10 \% \mathrm{RH}\right)$ increased rapidly above the $\mathrm{PV}$ of the oil sample stored under condition $1\left(60^{\circ} \mathrm{C}, 40 \% \mathrm{RH}\right)$. That is, the PV was generally stabilized during the four days at less than $4.2 \mathrm{meq} / \mathrm{kg}$ at a $60^{\circ} \mathrm{C}$, whereas the observed PV of the oil sample stored at the higher temperature of $80^{\circ} \mathrm{C}$ increased rapidly until it reached $10.3 \mathrm{meq} / \mathrm{kg}$ on the same fourth day. Moreover, the PV of the oil sample stored at the higher temperature of $80^{\circ} \mathrm{C}$ condition was measured as $14 \mathrm{meq} / \mathrm{kg}$ on the sixth day. Based on a previous study, which reported that rancid tastes and odors are clearly noticeable when the PV exceeds $20 \mathrm{meq} / \mathrm{kg}$ [27], we assume that the perilla seed oil sample can be significantly degraded within six days by hightemperature conditions $80^{\circ} \mathrm{C}$, as suggested in this study.

These results indicate that the free fatty acids that were produced by the oil oxidation process can be more easily affected by relative humidity than temperature, whereas the peroxides that were formed by iodine released from potassium iodide by an oil oxidation process were more significantly affected by temperature than relative humidity during the initial and intermediate processes of the oil oxidation under the storage environments in this study. Similarly to our study results, previous studies showed that the oxidation process of oil stored at around $60^{\circ} \mathrm{C}$ was significantly affected by both the moisture content of the 
TABLE 3: Results of PCR calibration and validation for acid and peroxide values of perilla seed oil according to preprocessing methods.

\begin{tabular}{|c|c|c|c|c|c|c|c|c|}
\hline \multirow{3}{*}{ Preprocessing methods } & \multicolumn{4}{|c|}{ Acid value } & \multicolumn{4}{|c|}{ Peroxide value } \\
\hline & \multicolumn{2}{|c|}{ Calibration } & \multicolumn{2}{|c|}{ Validation } & \multicolumn{2}{|c|}{ Calibration } & \multicolumn{2}{|c|}{ Validation } \\
\hline & $R^{2}$ & RMSE & $R^{2}$ & RMSE & $R^{2}$ & RMSE & $R^{2}$ & RMSE \\
\hline Raw data & 0.8979 & 0.0699 & 0.6501 & 0.1293 & 0.8240 & 1.6833 & 0.7748 & 1.9136 \\
\hline MSC & 0.8645 & 0.0805 & 0.7091 & 0.1179 & 0.8787 & 1.4042 & 0.7328 & 2.0841 \\
\hline SNV & 0.8373 & 0.0882 & 0.6859 & 0.1225 & 0.8120 & 1.7484 & 0.7552 & 1.9951 \\
\hline \multicolumn{9}{|l|}{ Normalization } \\
\hline Max & 0.8390 & 0.0877 & 0.5344 & 0.1492 & 0.8403 & 1.6113 & 0.7654 & 1.9529 \\
\hline Mean & 0.8785 & 0.0762 & 0.6985 & 0.1201 & 0.8575 & 1.5156 & 0.7833 & 1.8771 \\
\hline Range & 0.8105 & 0.0952 & 0.6454 & 0.1302 & 0.8157 & 1.7308 & 0.7474 & 2.0265 \\
\hline
\end{tabular}

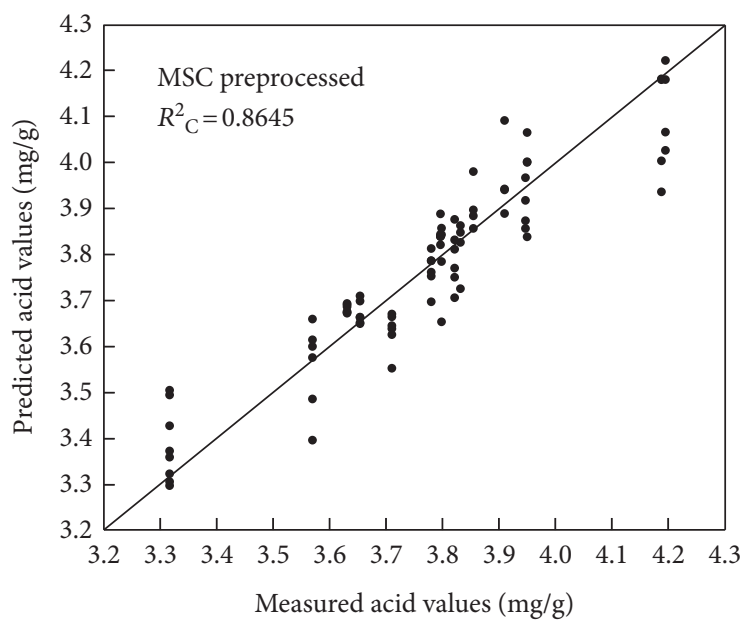

(a)

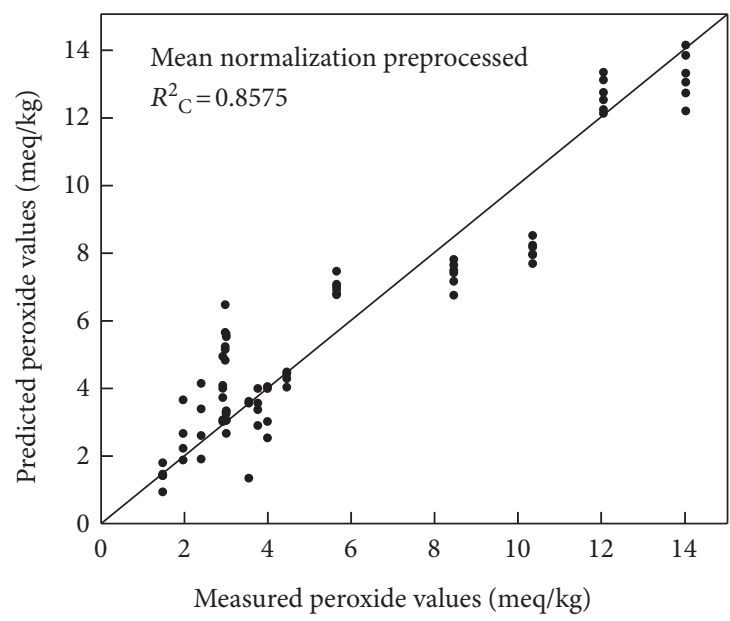

(c)

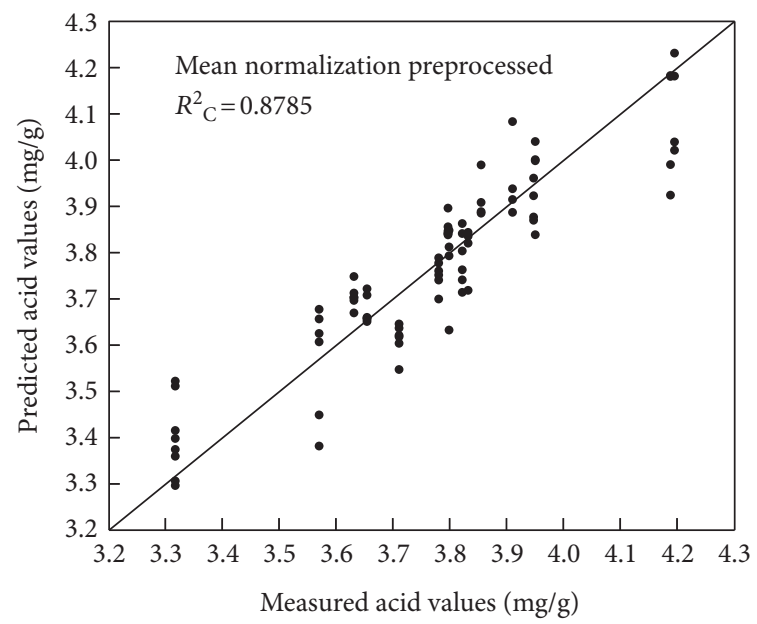

(b)

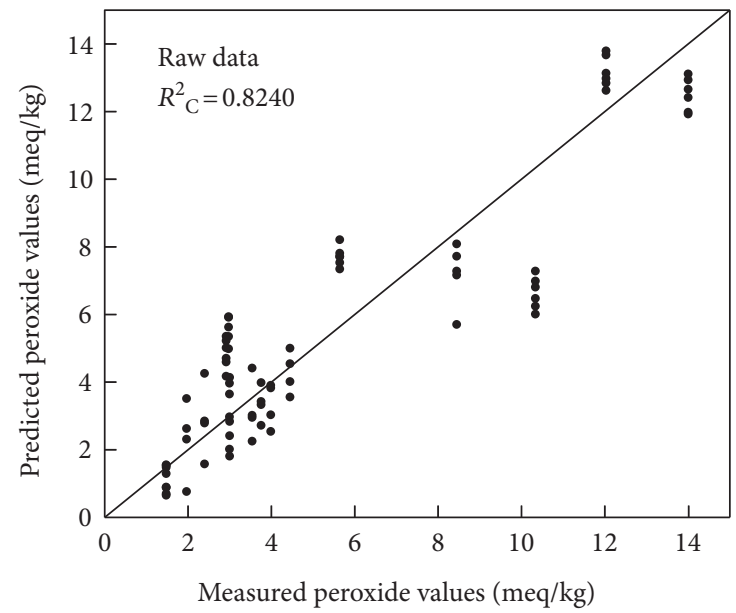

(d)

FIGURE 5: Prediction results of PCR models for acid and peroxide values from the highest $R^{2}$ values. (a) Acid value with MSC preprocessed. (b) Acid value with mean normalization. (c) Peroxide value with mean normalization. (d) Peroxide value with raw data.

oil itself and the relative humidity of the storage environment [28-31].

3.2. Transmittance Spectra of Perilla Seed Oil. Figure 4 shows the measured transmittance spectra of the oil samples over the storage period. As the storage period increases, the spectral absorption in the near-infrared band gradually increases on the whole, and large absorption peak can be observed at around wavelengths of 1200 and $1400 \mathrm{~nm}$. Generally, fatty acids in vegetable oils like perilla seed oil contain about $60 \% \alpha$-linolenic acid, which is a highly unsaturated fatty acid known as omega 3 and which is prone to induce rancidity due to the storage environment (temperature and moisture). It is known that hydroperoxide and carbonyl compounds, 
TABLE 4: Results of PLSR calibration and validation for acid and peroxide values of perilla seed oil according to preprocessing methods.

\begin{tabular}{|c|c|c|c|c|c|c|c|c|}
\hline \multirow{3}{*}{ Preprocessing methods } & \multicolumn{4}{|c|}{ Acid value } & \multicolumn{4}{|c|}{ Peroxide value } \\
\hline & \multicolumn{2}{|c|}{ Calibration } & \multicolumn{2}{|c|}{ Validation } & \multicolumn{2}{|c|}{ Calibration } & \multicolumn{2}{|c|}{ Validation } \\
\hline & $R^{2}$ & RMSE & $R^{2}$ & RMSE & $R^{2}$ & RMSE & $R^{2}$ & RMSE \\
\hline Raw data & 0.8257 & 0.0913 & 0.6887 & 0.1220 & 0.8209 & 1.7061 & 0.8036 & 1.7869 \\
\hline MSC & 0.8643 & 0.0806 & 0.7369 & 0.1122 & 0.8255 & 1.6842 & 0.7858 & 1.8660 \\
\hline SNV & 0.8703 & 0.0787 & 0.7272 & 0.1142 & 0.8268 & 1.6781 & 0.7905 & 1.8455 \\
\hline \multicolumn{9}{|l|}{ Normalization } \\
\hline Max & 0.8741 & 0.0776 & 0.7225 & 0.1152 & 0.8340 & 1.6428 & 0.7914 & 1.8416 \\
\hline Mean & 0.8328 & 0.0894 & 0.6681 & 0.1260 & 0.8037 & 1.7863 & 0.7816 & 1.8844 \\
\hline Range & 0.9163 & 0.0633 & 0.7413 & 0.1112 & 0.8460 & 1.5821 & 0.7829 & 1.8787 \\
\hline
\end{tabular}

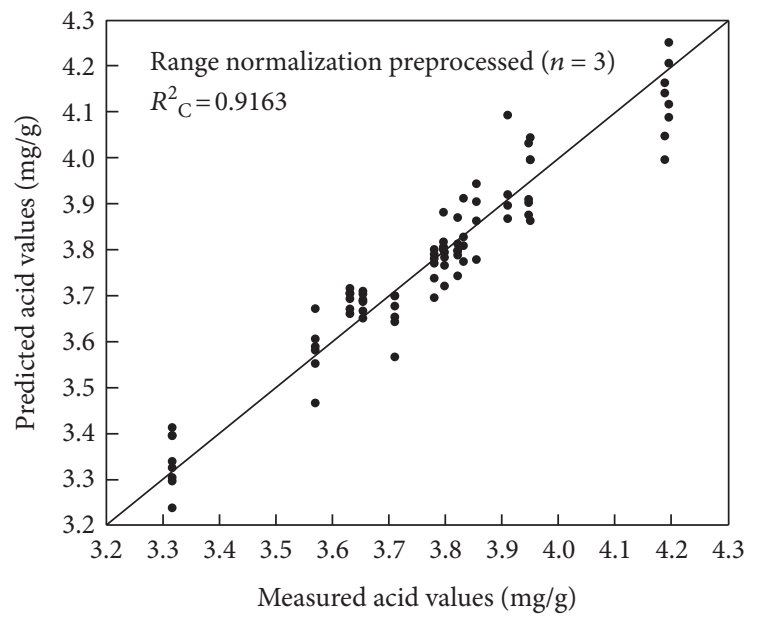

(a)

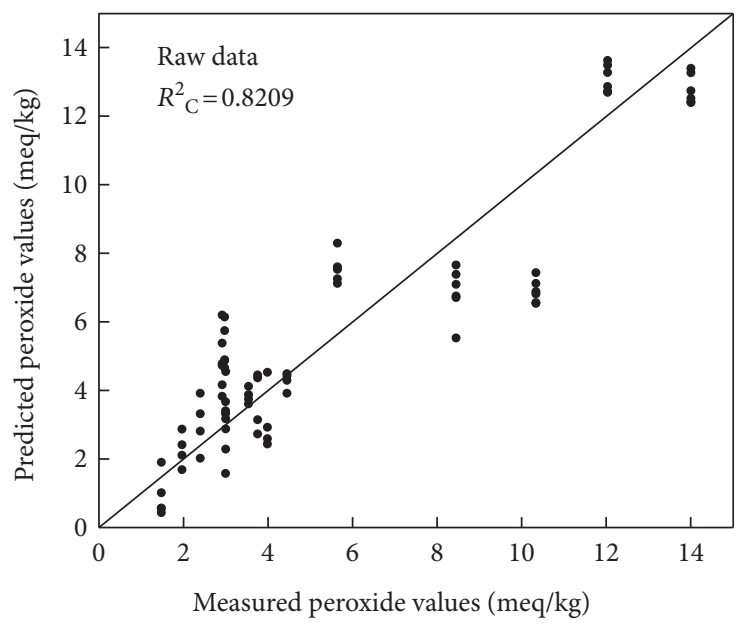

(c)

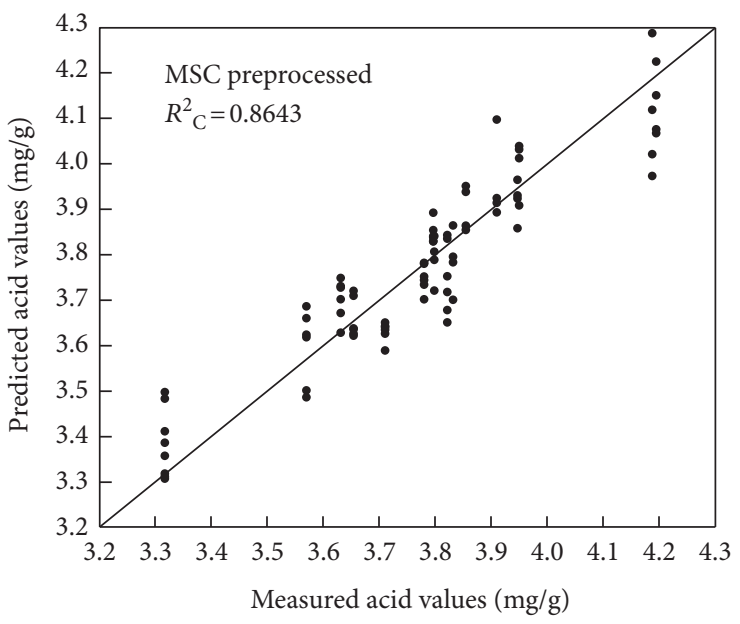

(b)

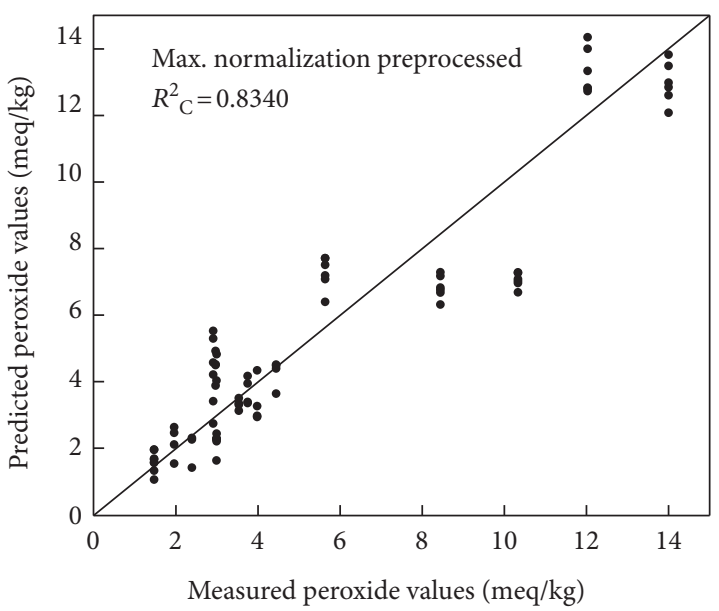

(d)

FIgURE 6: Prediction results of PLSR models for acid and peroxide values from the highest $R^{2}$ values. (a) Acid value with range normalization $(n=3)$. (b) Acid value with MSC preprocessed. (c) Peroxide value with raw data. (d) Peroxide value with maximum normalization.

which are not found in fresh vegetable oil (perilla seed oil), can be formed and cause an increase in the $-\mathrm{ROOH},-\mathrm{OH}$, $=\mathrm{O}$, and $-\mathrm{CHO}$ groups. Table 2 shows the commonly known wavelength locations of function groups and corresponding vibration modes which can be activated in the near-infrared region, and this information demonstrates the results of large spectral absorption at around the wavelength of
1200 and $1400 \mathrm{~nm}$ when the rancidity of vegetable oil induces the increasing acid value and peroxide value as shown in Figure 4.

3.3. Results of Principal Component Regression. The performances of PCR models for determining the AVs and PVs of the oil samples are summarized in Table 3 by showing 
TABLE 5: Performance of ANN models for acid value predictions.

\begin{tabular}{lccccccccc}
\hline \multirow{2}{*}{ Nodes } & \multicolumn{3}{c}{ Training } & \multicolumn{3}{c}{ Validation } & \multicolumn{3}{c}{ Test } \\
& $R^{2}$ & RMSE & Elapsed time (sec) & $R^{2}$ & RMSE & Elapsed time (sec) & $R^{2}$ & RMSE & Elapsed time (sec) \\
\hline 50 & 0.8440 & 0.0847 & 1 & 0.6001 & 0.1586 & 1 & 0.6764 & 0.1177 \\
70 & 0.8942 & 0.0745 & 1 & 0.7728 & 0.1008 & 1 & 0.6572 & 0.1181 \\
90 & 0.9037 & 0.0694 & 1 & 0.8175 & 0.0963 & 1 & 0.8555 & 0.1112 \\
110 & 0.9374 & 0.0611 & 2 & 0.8393 & 0.0779 & 2 & 0.6777 & 0.0935 \\
\hline
\end{tabular}

TABLE 6: Performance of ANN models for peroxide value predictions.

\begin{tabular}{lccccccccc}
\hline \multirow{2}{*}{ Nodes } & \multicolumn{3}{c}{ Training } & \multicolumn{2}{c}{ Validation } & \multicolumn{3}{c}{ Test } \\
& $R^{2}$ & RMSE & Elapsed time $(\mathrm{sec})$ & $R^{2}$ & RMSE & Elapsed time (sec) & $R^{2}$ & RMSE & Elapsed time (sec) \\
\hline 50 & 0.8439 & 1.5760 & 1 & 0.8266 & 1.9282 & 1 & 0.6458 & 2.1710 \\
70 & 0.9388 & 0.9862 & 1 & 0.8304 & 1.6262 & 1 & 0.8140 & 2.0883 \\
90 & 0.9210 & 1.1986 & 1 & 0.9341 & 0.9806 & 1 & 0.8286 & 1.5867 \\
110 & 0.9195 & 1.1220 & 2 & 0.8224 & 1.8588 & 2 & 0.8159 & 1.7379 \\
\hline
\end{tabular}

the coefficients of determination and RMSE values. Based on the validation results of the regression models for the AV, the MSC model shows the highest performance $\left(R_{\mathrm{C}}^{2}: 0.8645, \mathrm{RMSE}_{\mathrm{C}}: 0.0805 ; R_{\mathrm{V}}^{2}: 0.7091, \mathrm{RMSE}_{\mathrm{V}}: 0.1179\right)$, and the performance of the model with mean normalization preprocessing was estimated as the second best $\left(R_{C}^{2}: 0.8785\right.$, $\left.\operatorname{RMSE}_{\mathrm{C}}: 0.0762 ; R_{\mathrm{V}}^{2}: 0.6985, \mathrm{RMSE}_{\mathrm{V}}: 0.1201\right)$. As for the validation results of the regression models for the PV, the performance of the model with mean normalization preprocessing was analyzed as the highest $\left(R^{2}: 0.8575, \mathrm{RMSE}_{\mathrm{C}}\right.$ : $1.5156 ; R_{\mathrm{V}}^{2}$ : $\left.0.7833, \mathrm{RMSE}_{\mathrm{V}}: 1.8771\right)$, and the model with raw data shows the second best performance $\left(R_{C}^{2}: 0.8240\right.$, $\left.\mathrm{RMSE}_{\mathrm{C}}: 1.6833 ; R_{\mathrm{V}}^{2}: 0.7748, \mathrm{RMSE}_{\mathrm{V}}: 1.9136\right)$. The loading matrix $P$, score matrix $T$, and beta matrix which are calculated during the PCR are summarized in Tables S1 available online at https://doi.org/10.1155/2017/1082612, S2, and S3, respectively. Figure 5 shows the prediction results of the two most highly correlated PCR models for acid and peroxide values.

3.4. Results of Partial Least Squares Regression (PLSR). Table 4 shows the performance of PLSR models for determining the acid and peroxide values of the oil samples according to preprocessing methods. The performance of each PLSR model was compared and evaluated by using the coefficient of determination and the RMSE value. On the basis of the validation results for the developed models, prediction model of AV with the range normalization preprocessing method shows the best performance $\left(R^{2}{ }_{C}: 0.9163\right.$, $\left.\mathrm{RMSE}_{\mathrm{C}}: 0.0633 ; R_{\mathrm{V}}^{2}: 0.7413, \mathrm{RMSE}_{\mathrm{V}}: 0.1122\right)$, and the performance of the MSC model was estimated as the second best $\left(R_{\mathrm{C}}^{2}: 0.8643, \mathrm{RMSE}_{\mathrm{C}}: 0.0806 ; R_{\mathrm{V}}^{2}: 0.7369, \mathrm{RMSE}_{\mathrm{V}}: 0.1122\right)$. As for the validation results of regression models for the $\mathrm{PV}$, the performance of the model with raw data was analyzed as the best $\left(R_{\mathrm{C}}^{2}: 0.8209, \mathrm{RMSE}_{\mathrm{C}}: 1.7061 ; R_{\mathrm{V}}^{2}: 0.8036\right.$, $\left.\mathrm{RMSE}_{\mathrm{V}}: 1.7869\right)$, and the model using maximum normalization shows the second best performance $\left(R_{C}^{2}: 0.8340\right.$, $\left.\mathrm{RMSE}_{\mathrm{C}}: 1.6428, R_{\mathrm{V}}^{2}: 0.7914, \mathrm{RMSE}_{\mathrm{V}}: 1.8416\right)$. In this PLSR process, the number of principle components was decided as five and each spectrum has 804 wavelengths. The loading matrices $P$ and $Q$, score matrix $T$, and beta matrix are summarized in Tables S4, S5, S6, and S7, respectively. Figure 6 shows the prediction result of two most highly correlated PLSR models for acid and peroxide values.

3.5. Results of ANN Models. Tables 5 and 6 represent the prediction performances of ANN models for the acid and peroxide values of perilla seed oils. Four different ANN models were developed by applying four different node numbers to the models, and the performance of each model was evaluated by using the coefficient of determination and RMSE value. Based on the test results of the ANN models, the prediction performance for acid values $\left(R_{\text {tra }}^{2}\right.$ : 0.9037, RMSE $\mathrm{tra}_{\text {tra }}: 0.0694 ; R_{\mathrm{val}}^{2}: 0.8175, \mathrm{RMSE}_{\mathrm{val}}: 0.0963$; $R_{\text {test }}^{2}: 0.8555$, RMSE test $_{0}: 0.1112$ ) was evaluated to be slightly better than that for peroxide values $\left(R_{\text {tra }}^{2}: 0.9210\right.$, $\mathrm{RMSE}_{\text {tra }}: 1.1986 ; R_{\mathrm{val}}^{2}: 0.9341, \mathrm{RMSE}_{\mathrm{val}}: 0.9806 ; R_{\text {test }}^{2}$ : $\left.0.8286, \mathrm{RMSE}_{\text {test }}: 1.5867\right)$. Figure 7 shows the prediction results of most highly correlated ANN models for acid and peroxide values; the number of hidden nodes was set to 90 .

\section{Conclusions}

In this study, we developed prediction models and estimated the acid and peroxide values of perilla seed oil to nondestructively estimate the rancidity in conjunction with the storage conditions by using NIR spectroscopy and multivariate analysis methods. These methods have some merits in reducing time-consuming repetitive experiments and supporting reliable results by minimizing the errors that may originated from the operator's skills. It is generally known that the NIR spectroscopy technique we used in the study can precisely and quantitatively estimate the optical response of functional groups for a target molecular structure through multivariate analysis methods. We employed several multivariate analysis methods, such as PCR, PLSR, and ANN, 


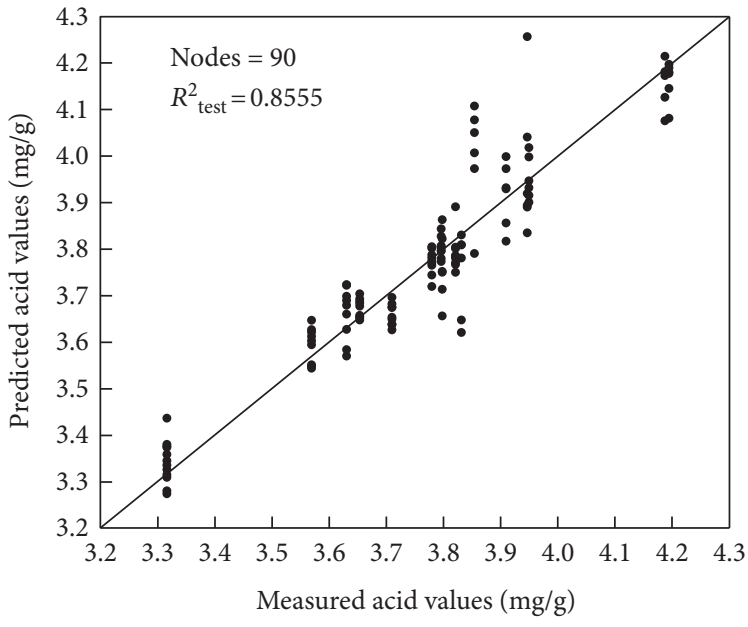

(a)

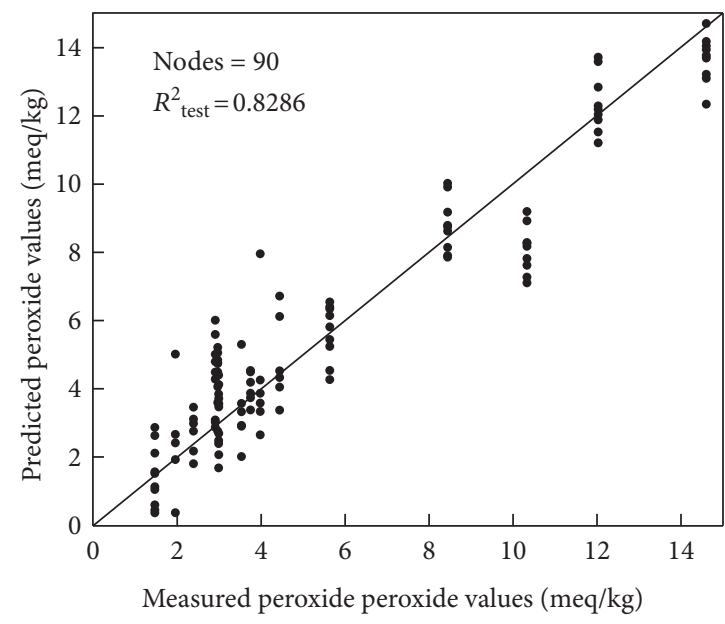

(b)

Figure 7: Graphs of the best results for the prediction of acid and peroxide values (90 nodes). (a) Acid value. (b) Peroxide value.

and it was determined that the prediction results for ANN models for both the acid value $\left(R_{\text {tra }}^{2}: 0.9037, R_{\text {val }}^{2}: 0.8175\right.$, and $\left.R_{\text {test }}^{2}: 0.8555\right)$ and peroxide value $\left(R_{\text {tra }}^{2}: 0.9210, R_{\text {val }}^{2}\right.$ : 0.9341 , and $R_{\text {test }}^{2}: 0.8286$ ) were the highest among the developed models.

The NIR spectroscopy technique used in this study has been commonly used in both qualitative and quantitative analyses of target samples by considering the vibrational energies of molecules, and the low optical absorbance characteristics of near-infrared rays facilitate deeper penetration than those of mid-infrared rays. In spite of its merits, NIR spectroscopy has limited ability to resolve noise, which is induced from measurement environments and the overlap tendencies of NIR spectra. A great deal of research is being performed in order to employ possible preprocessing methods and develop proper multivariate analysis models. We expect that our application of the PCR, PLSR, and ANN multivariate analysis methods for the nondestructive evaluation of the rancidity of perilla seed oil by using NIR spectroscopy techniques has great potential for use in the component analysis of agricultural products and foods, as well as many other scientific areas, such as life sciences and biomaterial researches.

\section{Conflicts of Interest}

The authors declare that they have no competing interests.

\section{Acknowledgments}

This work was supported by the National Research Foundation of Korea (Grant 2017R1D1A1A02019090).

\section{References}

[1] W. A. May, R. J. Peterson, and S. S. Chang, "Chemical reactions involved in the deep-fat frying of foods: IX. Identification of the volatile decomposition products of triolein," Journal of the American Oil Chemists' Society, vol. 60, no. 5, pp. 990995, 1983.
[2] I. H. Kim, S. Y. Jung, J. S. Jo, and Y. E. Kim, "Changes in components and sensory attribute of the oil extracted from perilla seed roasted at different roasting conditions," Agricultural Chemistry and Biotechnology, vol. 39, no. 2, pp. 118-122, 1996.

[3] Y. S. Cho, B. K. Kim, J. K. Park, J. W. Jeong, S. W. Jeong, and J. H. Lim, "Influence of thermal treatment on chemical changes in cold-pressed perilla seed oil," Korean Journal of Food Preservervation, vol. 16, no. 6, pp. 884-892, 2009.

[4] C. U. Choi, "History and science of perilla seed oil and sesame oil," Proceedings of the Korean Society of Food and Cookery Science Conference, vol. 37, no. 1, pp. 443-452, 1998.

[5] H. K. Chung, C. S. Choe, J. H. Lee, M. J. Chang, and M. H. Kang, "Oxidative stability of the pine needle extracted oils and sensory evaluation of savored laver made by extracted oils," Journal of the Korean Society of Food Culture, vol. 18, no. 2, pp. 89-95, 2003.

[6] F. W. Summerfield and A. L. Tappel, "Detection and measurement by high-performance liquid chromatography of malondialdehyde crosslinks in DNA," Analytical Biochemistry, vol. 143 , no. 2 , pp. $265-271,1984$.

[7] D. S. Kim, B. S. Koo, and M. S. Ahn, "A study on the formation of trans fatty acids with heating and storage of fats and oils (I) the change of physicochemical characteristics and total trans fatty acids content," Korean Journal of Food and Cookery Science, vol. 6, no. 2, pp. 37-50, 1990.

[8] N. R. Artman, "The chemical and biological properties of heated and oxidized fats," Advances in Lipid Research, vol. 7, pp. 245-330, 1969.

[9] S. Husain, G. S. R. Sastry, and N. P. Raju, "Molecular weight averages as criteria for quality assessment of heated oils and fats," Journal of the American Oil Chemists Society, vol. 68, no. 11, pp. 822-826, 1991.

[10] S. L. Taylor, C. M. Berg, N. H. Shoptaugh, and E. Traisman, "Mutagen formation in deep-fat fried foods as a function of frying conditions," Journal of the American Oil Chemists' Society, vol. 60, no. 3, pp. 576-580, 1983.

[11] E. Ben-Dor, J. R. Irons, and G. F. Epema, Remote Sensing for the Earth Sciences: Manual of Remote Sensing, John Wiley \& Sons, New York, Third edition, 1999. 
[12] E. Choe, F. van der Meer, F. van Ruitenbeek, H. van der Werff, B. de Smeth, and K. W. Kim, "Mapping of heavy metal pollution in stream sediments using combined geochemistry, field spectroscopy, and hyperspectral remote sensing: a case study of the Rodalquilar mining area, SE Spain," Remote Sensing of Environment, vol. 112, no. 7, pp. 3222-3233, 2008.

[13] C. H. Choi, K. J. Lee, and B. S. Park, "Prediction of soluble solid and firmness in apple by visible/near-infrared spectroscopy," Journal of the Korean Society for Agricultural Machinery, vol. 22, no. 2, pp. 256-265, 1997.

[14] R. F. Lu, D. E. Guyer, and R. M. Beaudry, "Determination of firmness and sugar content of apples using nearinfrared diffuse reflectance," Journal of Texture Studies, vol. 31, no. 6, pp. 615630,2000 .

[15] Y. T. Kim and S. R. Suh, "Comparison of performance of models to predict hardness of tomato using spectroscopic data of reflectance and transmittance," Journal of Biosystems Engineering, vol. 33, no. 1, pp. 63-68, 2008.

[16] S. Kawano, H. Watanabe, and M. Iwamoto, "Determination of sugar content in intact peaches by near infrared spectroscopy," Journal of Japanese Society for Horticultural Science, vol. 61, no. 2, pp. 445-451, 1992.

[17] G. G. Dull, R. G. Leffler, G. S. Birth, and D. A. Smittle, "Instrument for non-destructive measurement of soluble solids in honeydew melons," Transactions of the American Society of Agricultural Engineers, vol. 35, no. 2, pp. 735-737, 1992.

[18] H. I. Chung and H. J. Kim, "Near-infrared spectroscopy: principles," Analytical Science \& Technology, vol. 13, no. 1, pp. 138-151, 2000.

[19] M. J. Martelo-Vidal and M. Vázquez, "Application of artificial neural networks coupled to UV-VIS-NIR spectroscopy for the rapid quantification of wine compounds in aqueous mixtures," CyTA - Journal of Food, vol. 13, no. 1, pp. 32-39, 2015.

[20] C. Cimpoiu, V. Cristea, A. Hosu, M. Sandru, and L. Seserman, "Antioxidant activity prediction and classification of some teas using artificial neural networks," Food Chemistry, vol. 127, no. 3, pp. 1323-1328, 2011.

[21] M. Gestal, M. P. Gomez-Carracedo, J. M. Andrade et al., "Classification of apple beverages using artificial neural networks with previous variable selection," Analytica Chimica Acta, vol. 524, no. 1-2, pp. 225-234, 2004.

[22] A. Gori, C. Cevoli, A. Fabbri, M. F. Caboni, and G. Losi, "A rapid method to discriminate season of production and feeding regimen of butters based on infrared spectroscopy and artificial neural networks," Journal of Food Engineering, vol. 109, no. 3, pp. 525-530, 2012.

[23] AOCS, Official Methods and Recommended Practices of the American Oil Chemists' Society, method Te 1a-64, American Oil Chemists' Society, Champaign, IL, USA, 1977.

[24] AOAC, Official Methods of Analysis, Association of Official Agricultural Chemists, AOAC Press, Washington DC, 16th edition, 1995.

[25] H. Lawson, Common Chemical Reactions in Food Oils and Fats, Food Oils and Fats, CBS Publishers and Distributors, New Delhi, India, 1997.

[26] F. Shahidi and U. N. Wanasundara, Methods for Measuring Oxidative Rancidity in Fats and Oils. Food Lipids: Chemistry, Nutrition and Biotechnology, CRC Press, New York, Second edition, 2002.
[27] R. S. Kirk and R. Sawyer, Pearson's Composition and Analysis of Foods, Addison Wesley Longman, Harlow, UK, 9th edition, 1991.

[28] J. W. Park, J. Y. Kim, M. J. Kim, and J. H. Lee, "Evaluation of oxygen-limitation on lipid oxidation and moisture content in corn oil at elevated temperature," Journal of the American Oil Chemists' Society, vol. 91, no. 3, pp. 439-444, 2014.

[29] W. Chaiyasit, R. J. Elias, D. J. McClements, and E. A. Decker, "Role of physical structures in bulk oils on lipid oxidation," Critical Reviews in Food Science and Nutrition, vol. 47, no. 3, pp. 299-317, 2007.

[30] D. J. Mcclements and E. A. Decker, "Lipid oxidation in oil-inwater emulsions: impact of molecular environment on chemical reactions in heterogeneous food systems," Journal of Food Science, vol. 65, no. 8, pp. 1270-1282, 2000.

[31] K. Schwarz, S. W. Huang, J. B. German, B. Tiersch, J. Hartmann, and E. N. Frankel, "Activities of antioxidants are affected by colloidal properties of oil-in-water and water-in-oil emulsions and bulk oils," Journal of Agricultural and Food Chemistry, vol. 48, no. 10, pp. 4874-4882, 2000. 

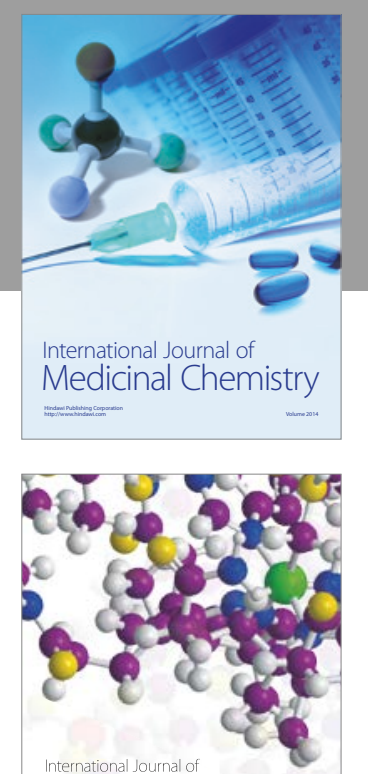

Carbohydrate Chemistry

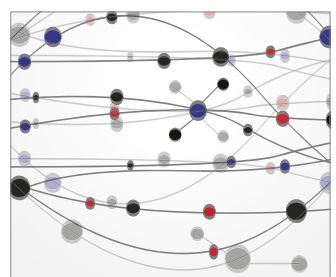

The Scientific World Journal
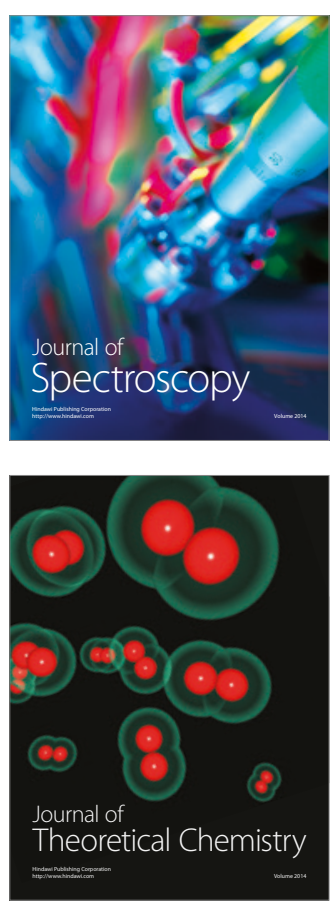
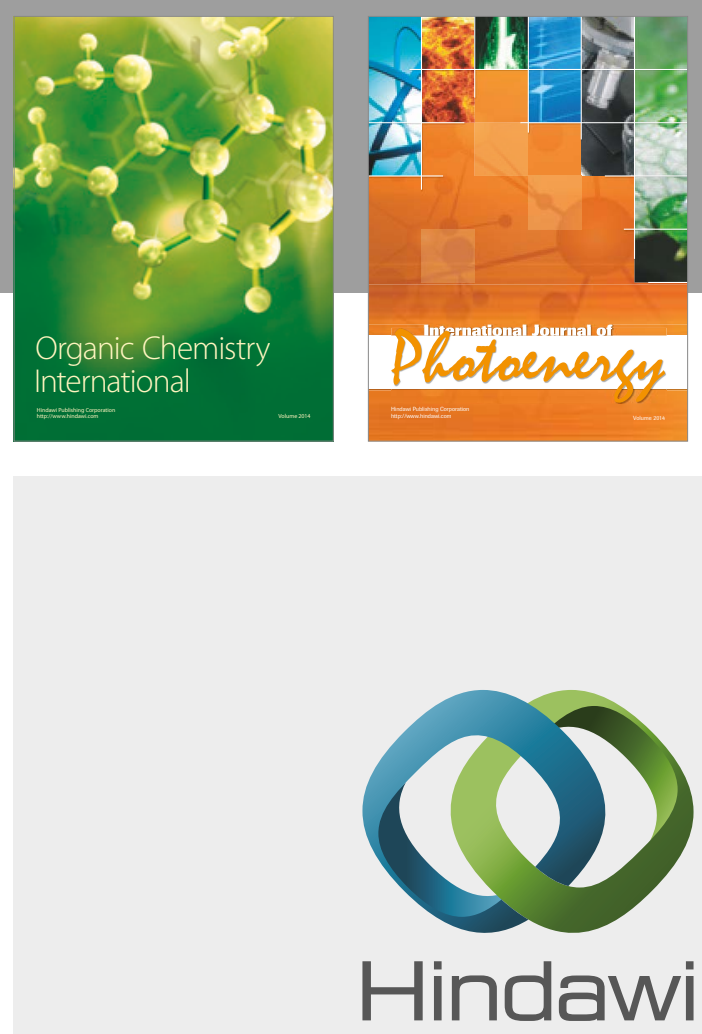

Submit your manuscripts at

https://www.hindawi.com

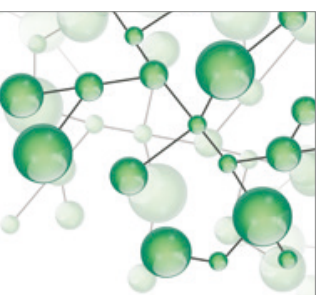

International Journal of

Inorganic Chemistry

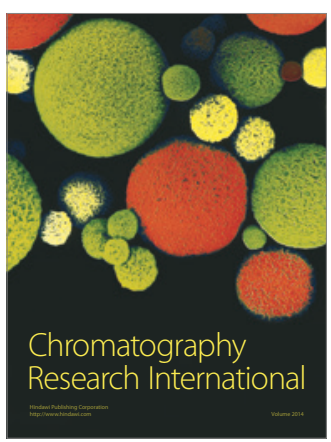

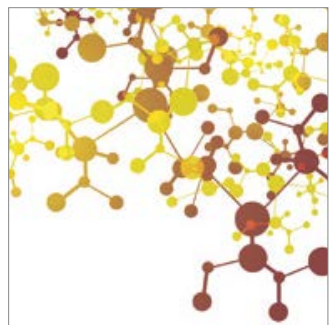

Applied Chemistry
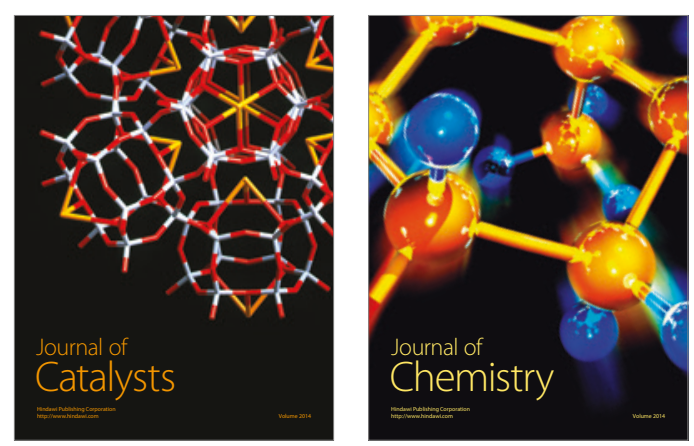
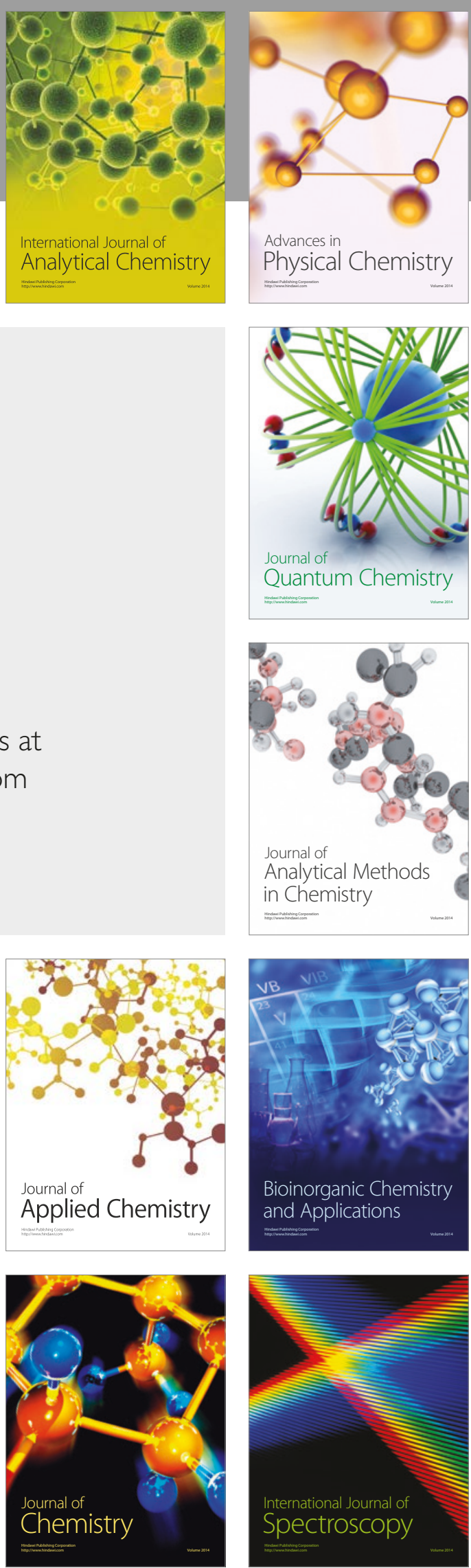